\title{
Propuesta de implementación de una empresa jardinera vertical, en la ciudad de Riobamba, Chimborazo - Ecuador
}

Proposal for the implementation of a vertical gardening company in the city of Riobamba, Chimborazo Ecuador

\author{
Marco Aníbal Vivar-Arrieta ${ }^{1 *}$ (D), María Fernanda Zumba-Mejía ${ }^{1}$
}

\section{RESUMEN}

La presente investigación se desarrolló para determinar la factibilidad de la creación de una empresa cuyo fin es el de implementar jardines verticales en las paredes externas e internas de las casas y edificios situados en la ciudad de Riobamba, provincia de Chimborazo, siendo el objetivo analizar el mercado potencial y determinar la factibilidad técnica, económica - financiera, mediante el método analítico. Se sectorizo la ciudad de Riobamba y se aplicaron encuestas a los ciudadanos de las casas y edificios dentro de la ciudad, a los cuales se les indagó sobre información de preferencias, demanda y complementariamente se analizó sobre proveedores, competidores, tipos de jardines verticales, plantas que serán utilizadas, costos para la empresa de jardinería vertical. Se establecieron costos y presupuestos para determinar la viabilidad económica y financiera, lo que permitió establecer que la empresa es viable con una inversión primaria de $\$ 92752$; con un VAN positivo, la empresa tendrá una tasa interna de retorno del $25 \%$ la cual es mayor a la tasa de descuento del $10 \%$ aplicada en el valor actual neto, concluyendo que la empresa tiene índice de beneficio costo de \$1,63 por lo que cada dólar invertido tendrá 63 centavos de ganancia.

Palabras claves: jardines verticales, Riobamba, factibilidad, viabilidad, económico-financiero.

\begin{abstract}
The present investigation was developed to determine the feasibility of creating a company whose purpose is to implement vertical gardens on the external and internal walls of houses and buildings located in the city of Riobamba, Chimborazo province, with the objective of analyzing the potential market and determine the technical feasibility, economic - financial, through the analytical method. The city of Riobamba was classified and surveys were applied to the citizens of the houses and buildings within the city, to which they were asked about information on preferences, demand and, in addition, suppliers, competitors, types of vertical gardens, plants were analyzed. which will be used, costs for the vertical gardening company. Costs and budgets were established to determine the economic and financial feasibility, which allowed to establish that the company is viable with a primary investment of $\$ 92,752$; with a positive NPV, the company will have an internal rate of return of $25 \%$ which is higher than the discount rate of $10 \%$ applied in the net present value, concluding that the company has a cost benefit index of $\$ 1.63$ per what each dollar invested will have 63 cents of profit.
\end{abstract}

Keywords: vertical gardens, Riobamba, feasibility, viability, economic-financial.

\footnotetext{
${ }^{1}$ Escuela Superior Politécnica de Chimborazo (ESPOCH), Panamericana Sur Km 1 1 1/2 entre Av. Canónigo Ramos y Av. 11 de noviembre, Chimborazo, Ecuador.

*Autor de Correspondencia, e-mail: marcovivar6@hotmail.com
} 


\section{INTRODUCCIÓN}

Actualmente el crecimiento demográfico de las ciudades acarea problemas tanto para el medio ambiente, salud y la calidad de vida de los pobladores. Por lo que esto incide en el incremento de procesos económicos esto se ve reflejado en las nuevas compañías, generando mayores emisiones de contaminantes (aire, agua), un fenómeno que se encuentra en constante crecimiento (Córdova 2015).

Por lo que surgen ideas sobre la importancia y preservación del paisaje natural y áreas verdes en la arquitectura, pero actualmente los espacios continúan siendo pequeños debido al crecimiento demográfico y urbanístico, ocasionando que las familias pierdan espacios verdes y plantas de diversos tipos que son agradables, los cuales sin duda mejoran la calidad de vida (Sanz y Vargas 2012).

Según Hasan (2013), La presencia de jardines en las vías y patios ajardinados, pero sobre todo de techos y fachadas ajardinados, podrían mejorar decididamente el clima polucionado de las ciudades: el aire se purificaría, se reducirían considerablemente los remolinos de polvo y las variaciones de temperatura y los porcentajes de humedad disminuirían.

Es por esto que los jardines verticales, partiendo de la necesidad de adaptar y mitigar los efectos del urbanismo y la contaminación, considerando la eco planificación como un pilar fundamental en la lucha contra la variación del clima (Minke 2012). Esto se debe al crecimiento urbano acelerado no planificado, provoca que las áreas verdes dentro de las ciudades disminuyan, entre ellas Riobamba, ocasionando impactos negativos en la calidad de vida de los pobladores, el medio ambiente y el paisaje por lo que surge la necesidad de aumentar espacios verdes, teniendo como una alternativa la jardinería vertical, permitiendo transformar las fachadas de las edificaciones en lugares donde las plantas crecen de forma óptima, regenerar el paisaje y brindar alternativas a aquellos habitantes que demandan productos como; flores, plantas medicinales, ornamentales, entre otras, permitiendo mejorar el aspecto y aumentando el valor de las edificaciones.
Es por ello que el objetivo de esta investigación fue analizar el mercado potencial y determinar la factibilidad técnica, económica - financiera para la creación de la empresa de jardinería vertical.

\section{MATERIA Y MÉTODOS}

\section{Área de estudio}

Se realizó en la ciudad de Riobamba, cantón Riobamba, provincia de Chimborazo- Ecuador, se tomó en consideración las condiciones climatológicas como: la temperatura promedio: $13,4{ }^{\circ} \mathrm{C}$, la Precipitación media anual: $510 \mathrm{~mm} / \mathrm{año}$, la Humedad relativa: $62,06 \%$. Los datos fueron proporcionados por la Estación Meteorológica de la Escuela Superior Politécnica de Chimborazo en 2017.

Según la clasificación ecológica del Ministerio del Ambiente [MAE] (2012), en la ciudad de Riobamba nos encontramos a una altura de 2758 m.s.n.m., correspondiente a Montano Bajo.

\section{Tamaño de la Muestra}

Determinación de la población

Según el INEC (2015), y aplicando la tasa estimada de crecimiento poblacional anual del $1,26 \%$ para obtener los datos de la población del 2017 y 2018 del cantón Riobamba, da como resultado una cifra de 258.597 habitantes aproximadamente al año 2018, que representan el $49 \%$ de la población de la provincia de Chimborazo y el 1,56 \% de la población del país. El área urbana del cantón cuenta con 124.807 habitantes concentra el $70,01 \%$ de la población cantonal. En general las parroquias rurales tienen una población inferior a los 9.000 habitantes.

A partir de la Clasificación Ascendente Jerárquica $(\mathrm{CAH})$ realizada por el Gobierno Autónomo descentralizado del cantón Riobamba, donde se utilizó indicadores de más de treinta variables del Censo del 2010 y del SIN 2015 relacionados con el estado de ocupación y densificación poblacional; la ocupación por grupos de edad y proveniencia; las características de confort en función del estado de las viviendas y grado de hacinamiento; el acceso a los servicios de comunicación al interior de las viviendas; las 
coberturas de servicios públicos; y las distancias a los equipamientos públicos básicos se obtiene cinco clases de áreas homogéneas en suelo urbano y periférico con mayor diferenciación entre ellas y con un grado suficiente de segmentación que permiten entender el estado, funcionalidad y demandas de la estructura urbana central que permitirán estructurar la propuesta de la creación de una empresa de jardinería vertical.
Considerando los datos recolectados por el GADR, se podrá trabajar con plenitud en las áreas de clase 2, 3 y 4, de los 5 antes mencionados ya que los ingresos son medios y altos, además se antepone la ubicación en la ciudad, la función ambiental y el interés general particular, garantizando el derecho de todos los riobambeños a un hábitat seguro y saludable. En la tabla 1 se considera los datos estadísticos en cifras reales para delimitar la población (GADR, 2018).

Tabla 1. Área homogénicas para la presente investigación

\section{Clase Población total Densidad personas Edad de 0 a 14 Edad de 15 a $64 \quad$ Edad de 65 a más}

\begin{tabular}{cccccc}
\hline Clase 2 & 59826 & 62,72 & 29,74 & 63,55 & 6,71 \\
\hline Clase 3 & 52322 & 35,97 & 26,67 & 68,77 & 4,56 \\
\hline Clase 4 & 33848 & 71,90 & 22,75 & 65,81 & 11,44 \\
\hline
\end{tabular}

Analizando las 3 clases se tiene 146000 personas, a esta cantidad se le divide el número de integrantes por familia $(3,75=4)$ registrado en el INEC (2015), y nos da un aproximado del número de casas y edificios factibles para la propuesta de jardinería. Entonces la sectorización define en un número de 36.500 usuarios potenciales de casas y edificios dentro de la ciudad de Riobamba.

\section{Determinación de la técnica y modelo estadístico para el cálculo de la muestra}

La técnica para levantar la información fue la encuesta ya que cada pregunta planteada fue de importancia para conseguir información concluyente para el desarrollo del estudio, constituida de 15 preguntas. Se utilizó el modelo estadístico de Barojas (2005) adecuado para determinar el tamaño de la muestra para poblaciones finitas, donde se utilizó un intervalo de confianza del $95 \%$, un nivel de confianza de $\mathrm{z}=1,96$; una desviación estándar de $\sigma=0,5$; un error de e $=$ $4,93 \%$; y unas probabilidades de $\mathrm{p}=0,5 ; \mathrm{q}=0,5$.

$$
\boldsymbol{n}=\frac{z^{2} * N * p * q}{e^{2} *(N-1)+z^{2} * p * q}
$$

Reemplazando se tiene:

$$
\begin{gathered}
n=\frac{(1,96)^{2} * 36500 * 0,5 * 0,5}{0,0493^{2} *(36500-1)+(1,96)^{2} * 0,5 * 0,5} \\
n=390
\end{gathered}
$$

\section{Recolección de información secundaria}

Se realizó un análisis de la industria el cual se desgloso en el análisis del entorno, permitiendo establecer mediante el producto interno bruto el crecimiento en la economía ecuatoriana, la actividad que presentó una mayor contribución a la variación del PIB y los competidores a nivel nacional. Concluyendo con el análisis del sector, que nos permitió conocer la zona de estudio, clientes, competidores a nivel local y la sostenibilidad en el tiempo de la empresa.

\section{Factibilidad Económica y Financiera}

En el análisis financiero se estableció los tipos de jardín vertical más demandados según el estudio de mercado, en función de esto se elaboró el flujo de caja, los costos de producción, el TIR, el VAN.

Se evaluó la factibilidad mediante los siguientes métodos:

\section{Valor Actual Neto (VAN)}

Rodrígues (2013), indica que la ecuación para calcular el VAN es la siguiente:

$\mathrm{VAN}=-\mathrm{I}+\frac{\mathrm{FC} 1}{(1+\mathrm{k})^{1}}+\frac{\mathrm{FC} 2}{(1+\mathrm{k})^{2}}+\cdots+\frac{\mathrm{FCn}}{(1+\mathrm{k})^{n}}=0$

Dónde:

I= Inversión Inicial

$\mathrm{FC}=$ Flujo de caja

$\mathrm{n}=$ Periodo de vida útil

$\mathrm{k}=$ Tasa de descuento 


\section{Tasa Interna de Retorno (TIR)}

De acuerdo con (Rodrígues, 2013), la ecuación para calcular la TIR es la siguiente:

$\operatorname{TIR}=-\mathrm{I}+\frac{\mathrm{FC} 1}{(1+\mathrm{r})^{1}}+\frac{\mathrm{FC} 2}{(1+\mathrm{r})^{2}}+\cdots+\frac{\mathrm{FCn}}{(1+\mathrm{r})^{n}}=0$

Dónde:

$\mathrm{I}=$ Inversión Inicial

$\mathrm{FC}=$ Flujo de caja

$\mathrm{n}=$ Periodo de vida útil

$\mathrm{r}=$ Tasa de descuento

\section{Relación beneficio-costo}

Se analizó el beneficio de la utilidad neta, sobre los costos que se tienen en el proyecto. Mediante la siguiente fórmula:

$I(b / c)=\frac{\sum \text { Flujo Acumulado de Fondos }}{\text { Inversión Inicial }}$

Dónde:

$\mathrm{I}(\mathrm{b} / \mathrm{c})=$ Relación beneficio-costo

\section{RESULTADOS}

\section{Análisis de la industria}

\section{Análisis del Entorno}

Según la figura 1, el Banco Mundial detalla al PIB como el producto interno bruto, siendo una medida macroeconómica que expresa el valor monetario de la producción de bienes y servicios de demanda final de un país o región durante un periodo de tiempo, normalmente se considera lapsos anuales. Es así que el progreso del PIB, en el Ecuador, se ha visto manifestado por una proporción creciente en los últimos años; específicamente las cifras son las siguientes:

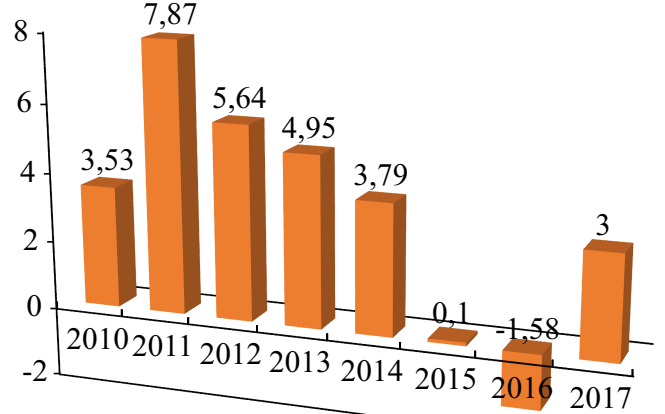

Figura 1. Variación del Producto Interno Bruto Ecuatoriano. Fuente: BCE, 2018

En la figura 1 se toma los valores del PIB desde el año 2010, registrando un valor máximo en el año 2011 a causa de un alto precio del petróleo, en los años posteriores se registra un decaimiento exponencial causado por los malos gobernantes y se tiene un decaimiento total del PIB en el año 2016 a causa del terremoto de grado 7,8 en la escala de Richter que azotó el país el 16 de abril del mismo año. La recuperación de la economía ecuatoriana (PIB) viene desde el año 2017, registrando un crecimiento en términos reales de 3,0\%. Este dinamismo se explica principalmente por el aumento del Gasto del Consumo Final de los Hogares, el Gasto de Consumo Final del Gobierno General y las Exportaciones. Este crecimiento representa el aumento de la renta o valor de bienes y servicios para una economía, en base a esto podemos afirmar que este crecimiento en la economía ecuatoriana es bastante favorable para el emprendimiento de nuevas empresas ya que a mayor crecimiento económico mayor demanda de bienes y servicios.

Según el Banco Central en su informe estadístico macroeconómico publicado para el segundo trimestre del año 2018 y que se encuentra en la figura 2, la actividad que presentó una mayor contribución a la variación positiva del PIB fue la construcción, siendo un componente importante al crecer constantemente la demanda de edificaciones, siendo un parámetro que nos favorece en la implementación de la empresa. 


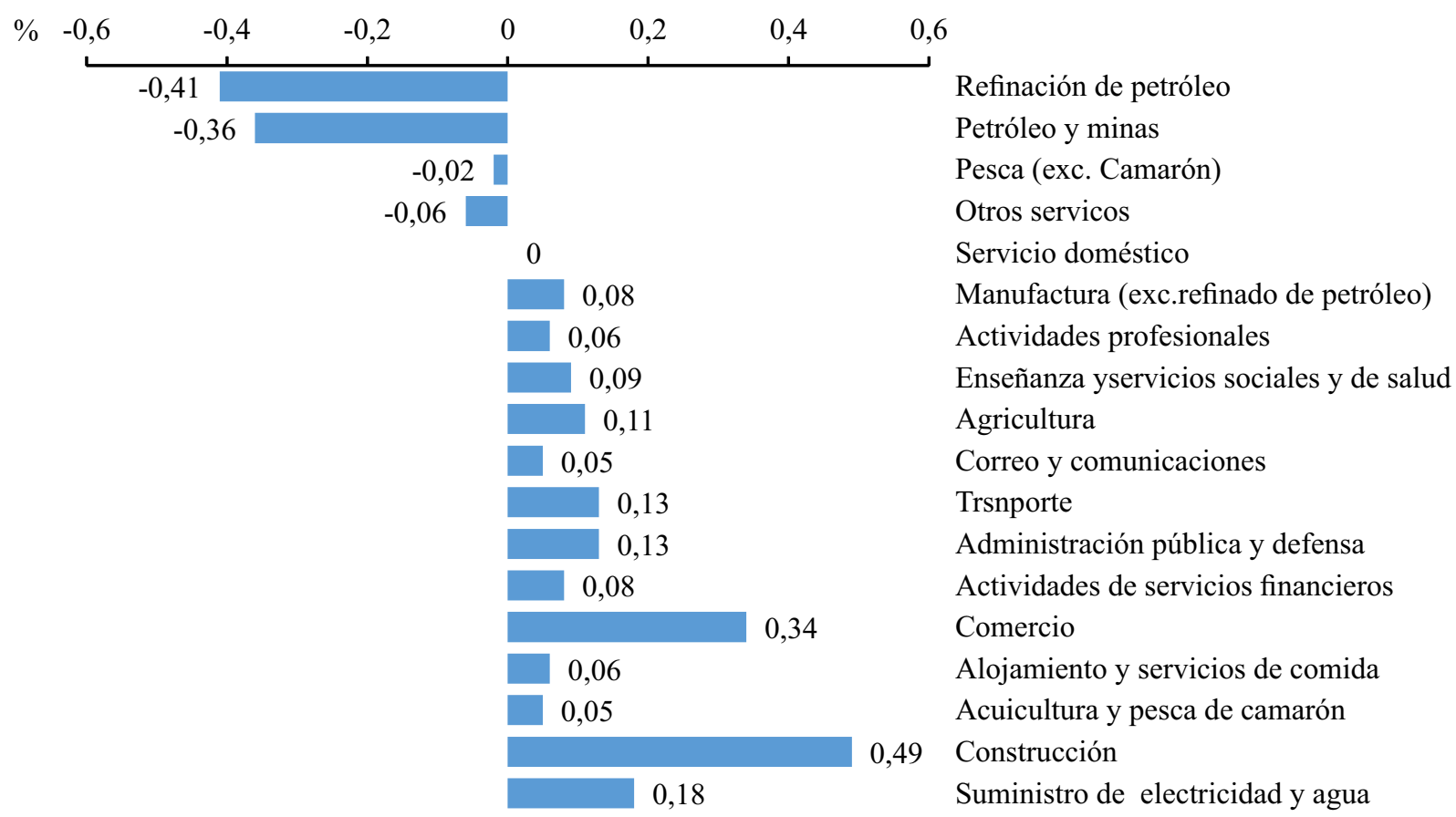

Figura 2. Contribución al crecimiento del PIB. Fuente: BCE, 2018.

Para corroborar las estadísticas mencionadas basta con caminar por las principales calles y avenidas de la ciudad de Riobamba y los alrededores para encontrar proyectos de vivienda, en especial construcciones verticales, así como propiedades en venta para estos fines. Un esclarecimiento para la gran acogida inmobiliaria en la Sultana de los Andes, se da porque hay más dinero en el mercado y la contribución del Banco del Instituto Ecuatoriano de Seguridad Social (BIESS).

Diferentes constructoras en la ciudad como Bienes Raíces Calderón, Bsl Constructora, Herrera Bienes Raíces, Vargas Arquitectos Riobamba, Covipal, Coa Inmobiliaria, Abitare Inmobiliaria y Urbec Constructora Inmobiliaria, afirman que cada año el crecimiento de la ciudad tiene un aumento constante, y cabe mencionar que una gran proporción de las construcciones son viviendas y edificaciones verticales, esto es un dato muy significativo ya que el estudio se encamina en la creación de jardines verticales en las paredes de casas y edificios, es así que se muestra un horizonte bastante bueno para el emprendimiento de la empresa que ofrezca este servicio.

\section{Análisis del Sector}

El sector de la jardinería en la ciudad de Riobamba está caracterizado por la prestación de servicios de manera informal por personas naturales, y no por empresas, sin embargo, existen empresas de otras ciudades que se dedican a las actividades de manutención, cuidado, plantación y arreglo de jardines, así como el diseño y construcción de los mismos. Específicamente, en esta rama, existen 4 empresas registradas en la Superintendencia de Compañías en la ciudad de Quito por lo que tendrían que viajar constantemente, (Césped Ecuador, Plantas y Jardines Ecuador, Pyrco y Bioparámetro) que están activas actualmente enero 2019.

Calificación del Área

\section{Clientes}

Los clientes pertenecen a la ciudad de Riobamba, dividido en clases sociales donde tienen ingresos medios y altos, además cuentan con casas y edificios aptos para la instalación de jardines verticales.

Proveedores

Uno de los proveedores directos será la empresa española "kahlapaisajismo" cuya sucursal se encuentra domiciliada en la ciudad de Quito, ya que, posee diseños únicos y variados, beneficiándonos de sus productos, e invención. Otros, son las empresas "El Agro y El Huerto", quienes ofrecen servicios de venta al por mayor y menor de plantas ornamentales, medicinales, frutales y hortícolas. 


\section{Competidores}

Nombre la empresa a establecerse JARDINES MILLENNIUM, no tendría contendientes directos en la ciudad, siendo los fundadores en la producción y mercantilización de jardinería vertical.

\section{Sostenibilidad en el tiempo}

Jardines Millennium se establecerá como una empresa fundadora de la implementación y comercialización de jardinería vertical en la ciudad y promoverá el cuidado del medio ambiente, paralelamente desarrollará modelos innovadores conforme a las demandas de los diferentes tipos de clientes.

\section{Análisis del mercado}

\section{Estudio de la demanda}

Para evitar un sobredimensionamiento del mercado se realizó un análisis de las encuestas demuestra que, dentro de la población, el $37 \%$ tienen jardines, mientras que el $63 \%$ no tiene. Dentro de las personas que dijeron que no contaban con jardín en su domicilio el $90 \%$ le gustaría contar con jardines y tan solo el $10 \%$ no desea tener jardines en el domicilio. Partiendo de si los encuestados conocen sobre la jardinería vertical, el $87 \%$ desconocen sobre la jardinería vertical, y el $1 \%$ rechaza la idea. Del total de las 382 personas encuestadas el $74 \%$ manifestaron su conformidad para implementar un jardín vertical en su casa, el $13 \%$ en el conjunto residencial, el $13 \%$ en el departamento y la gente que vivía en casa arrendada manifestó que colocarían al momento de obtener su casa propia.

Finalmente, de la totalidad de las personas encuestadas que les gustaría tener jardines verticales el $98 \%$ indica que estaría dispuesto a contratar el servicio de una empresa de jardinería vertical en la ciudad de Riobamba, mientras que el $2 \%$ indican que no estaría dispuesto a contratar el servicio.

Determinación de la demanda (población)

Considerando los datos recolectados por el GADR y con los de la (Tabla 1) en donde se define en un número de 36500 usuarios potenciales de casas y edificios dentro de la ciudad de Riobamba. Finalmente podemos inferir que 34310 personas estarían dispuestas a contratar el servicio de jardinería vertical, mientras que un mínimo 2190 no estarían dispuestos según el nivel de aceptación del proyecto.

$\underline{\text { Proyección de la demanda }}$

La proyección de la demanda entrega el valor de los servicios que brindará la empresa y que el mercado estaría dispuesto a adquirir durante una perspectiva establecida. La tasa de crecimiento promedio de los últimos años del sector de la construcción en Ecuador, es alta lo cual beneficia al desarrollo de la industria. En la tabla 2, se encuentra, la proyección desde el año base 2018, mediante los datos recuperados en las encuestas, de ese valor se trabaja con el $0,4 \%$ de la población total y se proyecta el $8 \%$ de crecimiento anual, el cual es la media de crecimiento de los últimos años.

\section{Fijación y Políticas de Precios}

En un análisis por Philip Kotler, el precio es la cuantía de dinero que se recauda por un servicio. Para la elaboración de esta investigación se compiló los costos manejados por las empresas descritas anteriormente que brindan servicios de diseño, construcción y mantenimiento de jardines verticales, los mismos que se encuentran detallados en las tablas 3 y 4 .

Tabla 2. Proyección de la demanda

\begin{tabular}{ccccc}
\hline $\mathbf{2 0 1 8}$ & $\mathbf{2 0 1 9}$ & $\mathbf{2 0 2 0}$ & $\mathbf{2 0 2 1}$ & $\mathbf{2 0 2 2}$ \\
\hline 135 & 142 & 149 & 157 & 164 \\
\hline
\end{tabular}

Tabla 3. Descripción de precios por unidades Sistema Modular Complejo

\begin{tabular}{lccc}
\hline \multicolumn{1}{c}{ Descripción } & Unidad & Costo \$ & Costo Total\$ \\
\hline Sistema modular compuesto (4 módulos x 4 macetas) & 1 Pack & 82 & 82 \\
Plantas ornamentales, medicinales, hortalizas o frutales & 16 & 0,75 & 12 \\
Rack de soporte & 1 & 12 & 12 \\
Sistema Imperlastic + Polibrea+Super KAR & 1 & 11,60 & 13,60 \\
Mini tanque deAgua con Sustratos & $6,5 \mathrm{~L}$ & 1 & 6,5 \\
\hline Total & & & $\$ 126,10$ \\
\hline
\end{tabular}


Tabla 4. Descripción de precios por unidades Sistema Modular Simple

\begin{tabular}{lccc}
\hline \multicolumn{1}{c}{ Descripción } & Unidad & Costo\$ & Costo Total\$ \\
\hline Sistema modular simple(6 módulos x 4 macetas)+Sistema & & & \\
de Riego+ Temporizador(3 tiempos)+Bomba de & 1 Pack & 89,5 & 89,5 \\
Fertilizante & & & 18 \\
Plantas ornamentales, medicinales, hortalizas o frutales & 24 & 0,75 & 13,60 \\
Sistema Imperlastic + Polibrea+Super KAR & 1 & 11,60 & 5 \\
Sustratos & $1 \mathrm{kit}$ & 1 & $\$ 126,10$ \\
\hline Total & & & \\
\hline
\end{tabular}

El servicio de diseño y construcción por metro cuadrado de un jardín vertical (SMC o SMS), tiene un costo de $\$ 126,10$.

\section{Plan financiero}

El objetivo principal es demostrar la factibilidad financiera y establecer el presupuesto de inversiones para el punto de partida del proyecto.

Presupuesto de inversiones

El presupuesto de las inversiones determina la estimación monetaria que será precisa para efectuar las inver- siones primarias para el punto de partida de la empresa, y los consecuentes meses de operaciones, siendo crucial para la estimación financiera del proyecto.

Determinación de la inversión total inicial

Inversiones fijas

Las inversiones fijas están ligadas a la adquisición de activos fijos tangibles, y los enseres para comenzar la labor de la empresa, las mismas que se encuentran detalladas en la tabla 5 .

Tabla 5. Activos fijos de la empresa Jardines Millennium

\begin{tabular}{|c|c|c|c|}
\hline Descripción & Cantidad & Costo & Costo Total \\
\hline \multicolumn{4}{|c|}{ Infraestructura } \\
\hline Bodega & 1 & 50000,00 & 50000,00 \\
\hline \multicolumn{4}{|c|}{ Muebles y enseres } \\
\hline Archivador & 2 & 120,00 & 240,00 \\
\hline Botiquín & 1 & 40,00 & 40,00 \\
\hline Dispensador agua y café & 1 & 95,00 & 95,00 \\
\hline Escritorio & 1 & 240,00 & 240,00 \\
\hline Escritorio recepción & 1 & 190,00 & 190,00 \\
\hline Extintor de incendios & 1 & 33,00 & 33,00 \\
\hline Mesa centro recepción & 1 & 70,00 & 70,00 \\
\hline Mueble & 2 & 40,00 & 80,00 \\
\hline Silla Giratoria & 2 & 119,00 & 238,00 \\
\hline Sillas & 5 & 40,00 & 200,00 \\
\hline \multicolumn{4}{|c|}{ Equipos de oficina } \\
\hline Basurero & 2 & 20,00 & 40,00 \\
\hline Computador & 2 & 850,00 & 1700,00 \\
\hline Impresora multifuncional & 2 & 450,00 & 900,00 \\
\hline Proyector & 1 & 475,00 & 475,00 \\
\hline Router internet & 1 & 25,00 & 25,00 \\
\hline Teléfonos & 2 & 40,00 & 80,00 \\
\hline Lámpara & 2 & 30,00 & 60,00 \\
\hline Plantas & 2 & 12,00 & 24,00 \\
\hline \multicolumn{4}{|c|}{ Herramientas de trabajo } \\
\hline Baúl almacenamiento materiales & 1 & 300,00 & 300,00 \\
\hline Kit herramientas jardinería & 2 & 90,00 & 180,00 \\
\hline Kit herramientas construcción & 2 & 120,00 & 240,00 \\
\hline Sistema modular simple & 3 & 15,00 & 45,00 \\
\hline Sistema modular complejo & 3 & 10,00 & 30,00 \\
\hline \multicolumn{4}{|c|}{ Vehículos } \\
\hline Camioneta doble cabina & 1 & 22000,00 & 22000,00 \\
\hline Total & & & 77525,00 \\
\hline
\end{tabular}




\section{Activos Diferidos}

Dentro de los gastos de constitución se considera los requisitos legales necesarios y los permisos obligatorios para poner en marcha la empresa. Los detalles se encuentran en la tabla 6.

Tabla 6. Activos Diferidos

\begin{tabular}{cc}
\hline Concepto & Costo \\
\hline Notaría & 400,00 \\
Estatutos de constitución & 100,00 \\
Obtención del RUC & 0,00 \\
Permisos municipales y bomberos & 154,00 \\
Honorarios abogado & 200,00 \\
Total & 854,00 \\
\hline
\end{tabular}

Capital de trabajo

Para Jardines Millennium el cálculo del capital de trabajo, representa la determinación de la cantidad de capital para cubrir los gastos en suministros e insumos, pago de salarios directos o indirectos y los gastos de administración y ventas durante el primer mes de trabajo de la empresa. Los valores del capital de trabajo se encuentran detallados en la tabla 7.

Tabla 7. Capital de Trabajo

\begin{tabular}{cc}
\hline Denominación & Valor \\
\hline Mano de obra directa & 874,77 \\
Materiales directo & 13498,33 \\
Total & 14373,10 \\
\hline
\end{tabular}

Mano de obra directa

El personal que interviene directamente en la instalación de los jardines verticales, se encuentra detallado en la tabla 8.

Tabla 8. Mano de Obra Directa

\begin{tabular}{cccccccc}
\hline Denominación & Sueldo & $\mathbf{1 3}^{\text {er }}$ sueldo & $\mathbf{1 4}^{\text {to }}$ sueldo & $\begin{array}{c}\text { Fondos } \\
\text { reserva }\end{array}$ & Vacaciones & $\begin{array}{c}\text { IESS } \\
(\mathbf{1 2 , 1 5 \% )}\end{array}$ & $\begin{array}{c}\text { Total } \\
\text { mes }\end{array}$ \\
\hline Jardinero 1 & 390,00 & 32,50 & 32,83 & 0,00 & 0,00 & 47,39 & 437,39 \\
Jardinero 2 & 390,00 & 32,50 & 32,83 & 0,00 & 0,00 & 47,39 & 437,39 \\
\hline Total & & & & & $\mathbf{8 7 4 , 7 7}$ \\
\hline
\end{tabular}

Materiales directos

Son todos los materiales que pueden identificarse para la instalación de los sistemas modulares y se encuentran detallados en la tabla 9 .

Tabla 9. Materiales Directos

\begin{tabular}{ccccc}
\hline Denominación & Unidad & Cantidad & Valor unit & Valor total mensual \\
\hline SMC & pack & 390 & 82,00 & 2665,00 \\
SMS & pack & 910 & 89,50 & 6787,08 \\
Impermeabilización & kit & 1300 & 13,60 & 1473,33 \\
Plantas & $\mathrm{u}$ & 28080 & 0,75 & 1755,00 \\
Rack & $\mathrm{u}$ & 390 & 12,00 & 390,00 \\
Sustratos & kit & 910 & 2,86 & 216,67 \\
Agua con Sustratos & kit & 2535 & 1,00 & 211,25 \\
\hline Total & & & & $\mathbf{1 3 4 9 8 , 3 3}$
\end{tabular}

Una vez definidos los activos fijos y el capital de trabajo se establece la inversión total inicial pretendida para el estudio de factibilidad que se encuentra detallada en la tabla 10.

Tabla 10. Inversión Total Inicial

\begin{tabular}{lc}
\hline Inversión Total Inicial & \\
\hline Denominación & Valor \\
Activos fijos & 77525,00 \\
Activos diferidos & 854,00 \\
Capital de trabajo & 14373,10 \\
\hline Total & $\mathbf{9 2 7 5 2 , 1 0}$ \\
\hline
\end{tabular}

Fuentes de Financiamiento

Tabla 11. Fuentes de Financiamiento

\begin{tabular}{lcc}
\hline \multicolumn{3}{l}{ Fuentes de Financiamiento } \\
\hline Detalle & Inversión & Porcentaje \\
Inversión total & 92752,10 & $\mathbf{1 0 0} \%$ \\
Capital Propio & 72000,00 & $\mathbf{7 8 \%}$ \\
Financiamiento & 20752,10 & $\mathbf{2 2 \%}$ \\
\hline
\end{tabular}




\section{Capital Propio}

Al considerar el modelo legal de empresa unipersonal de responsabilidad limitada, el capital propio es por parte del Gerente propietario, no existe ningún tipo de accionista.

\section{Financiamiento}

Se considera a BanEcuador (Institución Financiera) como fuente de financiamiento para el estudio de factibilidad ya que la actividad a realizar consta dentro de las actividades financiables al requerir financiar solo el capital de trabajo. El préstamo se realizará con un plazo establecido a 3 años es el plazo máximo para financiamiento de capital de trabajo; por un monto total de $\$ 20753,00$ con una tasa efectiva del $11.83 \%$ anual.

Presupuesto de ventas

Considerando que los meses iniciales son de adecuación y lanzamiento se estableció un presupuesto men- sual de ventas en concordancia con la situación real, por el cual se pretende cubrir la demanda según el tiempo y la capacidad de trabajo ejecutable y además se estableció una proyección de crecimiento del $8 \%$ anual, el cual es la media de crecimiento de los últimos años presentada en la Tabla 12.

Tabla 12. Proyección de Instalación por $\mathrm{m}^{2}$ anual

\begin{tabular}{cccccc}
\hline Año & 2019 & 2020 & 2021 & 2022 & 2023 \\
\hline $\begin{array}{c}\text { Cantidad } \\
\text { de m }^{2} \\
\text { instalados }\end{array}$ & 1300 & 1400 & 1500 & 1600 & 1700 \\
\hline
\end{tabular}

Tomando en cuenta los valores definidos en las tablas 3 y 4, el valor monetario por instalación de un metro cuadrado es de $\$ 190,00$ con un incremento proyectado del 0,27\% anual, valor establecido para la inflación en Ecuador en el año 2018, las proyecciones de precios por instalación se encuentran en la tabla 13.

Tabla 13. Proyección de precios por instalación de un $\mathrm{m}^{2}$ anual

\begin{tabular}{llllll}
\hline Metros cuadrados & 1300 & 1400 & 1500 & 1600 & 1700 \\
Precio unitario & 190,00 & 190,51 & 191,03 & 191,54 & 192,06 \\
\hline Total & $\mathbf{2 4 7 0 0 0 , 0 0}$ & $\mathbf{2 6 6 7 1 8 , 2 0}$ & $\mathbf{2 8 6 5 4 1 , 0 8}$ & $\mathbf{3 0 6 4 6 9 , 0 5}$ & $\mathbf{3 2 6 5 0 2 , 5 5}$ \\
\hline
\end{tabular}

El presupuesto mensual detallado para el primer año y ajustando a la situación se encuentra en la tabla 14:

Tabla 14. Presupuesto de ventas mensual

\begin{tabular}{cccc}
\hline Mes & $\begin{array}{c}\mathbf{N}^{\circ} \\
\text { instalaciones } \\
\text { por m }\end{array}$ & P Uni & Total \\
\hline Enero & 90 & 190,00 & $\mathbf{1 7 1 0 0 , 0 0}$ \\
Febrero & 90 & 190,00 & $\mathbf{1 7 1 0 0 , 0 0}$ \\
Marzo & 90 & 190,00 & $\mathbf{1 7 1 0 0 , 0 0}$ \\
Abril & 100 & 190,00 & $\mathbf{1 9 0 0 0 , 0 0}$ \\
Mayo & 100 & 190,00 & $\mathbf{1 9 0 0 0 , 0 0}$ \\
Junio & 100 & 190,00 & $\mathbf{1 9 0 0 0 , 0 0}$ \\
Julio & 110 & 190,00 & $\mathbf{2 0 9 0 0 , 0 0}$ \\
Agosto & 110 & 190,00 & $\mathbf{2 0 9 0 0 , 0 0}$ \\
Septiembre & 110 & 190,00 & $\mathbf{2 0 9 0 0 , 0 0}$ \\
Octubre & 130 & 190,00 & $\mathbf{2 4 7 0 0 , 0 0}$ \\
Noviembre & 130 & 190,00 & $\mathbf{2 4 7 0 0 , 0 0}$ \\
Diciembre & 140 & 190,00 & $\mathbf{2 6 6 0 0 , 0 0}$ \\
\hline
\end{tabular}

Presupuesto de Costos

\section{Costo de materiales}

Tomando en cuenta que el tamaño promedio de los jardines a construir es de $138 \mathrm{~m}^{2}$. Proyectando los costos para los 5 años teniendo un incremento de inflación del $0,27 \%$, se establece el presupuesto de costos por $\mathrm{m}^{2}$ anual en la tabla 15 .
Tabla 15. Presupuesto de costos por $\mathrm{m}^{2}$ Anual

\begin{tabular}{cccc}
\hline Año & $\mathbf{N}^{\circ} \mathbf{m}^{\mathbf{2}}$ & P Uni & Total \\
\hline 2018 & 1300 & 190,00 & $\mathbf{2 4 7 0 0 0 , 0 0}$ \\
2019 & 1370 & 190,51 & $\mathbf{2 6 6 7 1 8 , 2 0}$ \\
2020 & 1430 & 191,03 & $\mathbf{2 8 6 5 4 1 , 0 8}$ \\
2021 & 1500 & 191,54 & $\mathbf{3 0 6 4 6 9 , 0 5}$ \\
2022 & 1580 & 192,06 & $\mathbf{3 2 6 5 0 2 , 5 5}$ \\
\hline
\end{tabular}

2. Costo de mano de obra

En las tablas 16 y 17, se exhiben los salarios establecidos para el personal administrativo y la mano de obra directa, considerando los beneficios de ley que se pagan pasado el primer año de servicio, es ineludible construir una proyección a 5 años para considerar todos los rubros; considerando un incremento en el sueldo básico de un $10 \%$ anual para mano de obra directa y $8 \%$ para mano de obra indirecta, el cual fue calculado en base al crecimiento salarial en el 2018 en Ecuador. 
Tabla 16. Mano de Obra Indirecta Anual

\begin{tabular}{cccccc}
\hline Denominación & $\mathbf{1}$ año & $\mathbf{2}$ año & $\mathbf{3}$ año & 4 año & 5 año \\
\hline Gerente propietario & 11542,97 & 12466,40 & 13463,72 & 14540,81 & 15704,08 \\
Recepcionista & 5313,95 & 5845,35 & 6429,88 & 7072,87 & 7780,16 \\
Diseñador de jardines & 6803,50 & 7347,78 & 7935,60 & 8570,45 & 9256,09 \\
Contador & 4366,06 & 4715,34 & 5092,57 & 5499,98 & 5939,98 \\
Agente de Limpieza & 5313,95 & 5845,35 & 6429,88 & 7072,87 & 7780,16 \\
\hline Total & $\mathbf{3 3 3 4 0 , 4 3}$ & $\mathbf{3 6 2 2 0 , 2 3}$ & $\mathbf{3 9 3 5 1 , 6 6}$ & $\mathbf{4 2 7 5 6 , 9 9}$ & $\mathbf{4 6 4 6 0 , 4 6}$ \\
\hline
\end{tabular}

Tabla 17. Mano de Obra Directa Anual

\begin{tabular}{|c|c|c|c|c|c|}
\hline Denominación & 1 año & 2 año & 3 año & 4 año & 5 año \\
\hline Jardinero 1 & 5313,95 & 5845,35 & 6429,88 & 7072,87 & 7780,16 \\
\hline Jardinero 2 & 5313,95 & 5845,35 & 6429,88 & 7072,87 & 7780,16 \\
\hline Total & 10627,91 & 11690,70 & 12859,77 & 14145,74 & 15560,32 \\
\hline
\end{tabular}

Gastos administrativos

Los gastos administrativos obligatorios son los servicios básicos de la oficina y la bodega, los suministros de oficina y los útiles de limpieza; proyectando los gastos a 5 años y se considera un incremento en los costos en un valor equivalente a la inflación de $0,27 \%$ y el detalle se encuentra en la tabla 18.

Tabla 18. Gastos administrativos anuales

\begin{tabular}{|c|c|c|c|c|c|}
\hline \multicolumn{6}{|c|}{ Servicios básicos anuales } \\
\hline Concepto & Año 1 & Año 2 & Año 3 & Año 4 & Año 5 \\
\hline Agua & 264,00 & 264,71 & 265,43 & 266,14 & 266,86 \\
\hline Luz & 720,00 & 721,94 & 723,89 & 725,85 & 727,81 \\
\hline Teléfono e Internet & 840,00 & 842,27 & 844,54 & 846,82 & 849,11 \\
\hline Total & 1824,00 & 1828,92 & 1833,86 & 1838,81 & 1843,78 \\
\hline \multicolumn{6}{|c|}{ Suministros y materiales anuales } \\
\hline Papelería & 120,00 & 120,32 & 120,65 & 120,97 & 121,30 \\
\hline Folders & 9,00 & 9,02 & 9,05 & 9,07 & 9,10 \\
\hline Caja de esferos & 78,00 & 78,21 & 78,42 & 78,63 & 78,85 \\
\hline Folder archivador & 180,00 & 180,49 & 180,97 & 181,46 & 181,95 \\
\hline Caja de grapas & 6,00 & 6,02 & 6,03 & 6,05 & 6,07 \\
\hline Caja de borrador & 36,00 & 36,10 & 36,19 & 36,29 & 36,39 \\
\hline Caja de lápices & 30,00 & 30,08 & 30,16 & 30,24 & 30,33 \\
\hline Marcador & 18,00 & 18,05 & 18,10 & 18,15 & 18,20 \\
\hline Caja clips & 24,00 & 24,06 & 24,13 & 24,19 & 24,26 \\
\hline Carpetas & 48,00 & 48,13 & 48,26 & 48,39 & 48,52 \\
\hline Total & 549,00 & 550,48 & $\mathbf{5 5 1 , 9 7}$ & 553,46 & 554,95 \\
\hline \multicolumn{6}{|c|}{ Equipo de limpieza anual } \\
\hline Toallas de limpieza & 36,00 & 36,10 & 36,19 & 36,29 & 36,39 \\
\hline Papel higiénico & 36,00 & 36,10 & 36,19 & 36,29 & 36,39 \\
\hline Cloro & 24,00 & 24,06 & 24,13 & 24,19 & 24,26 \\
\hline Gel antibacterial & 96,00 & 96,26 & 96,52 & 96,78 & 97,04 \\
\hline Jabón líquido & 84,00 & 84,23 & 84,45 & 84,68 & 84,91 \\
\hline Desinfectante de pisos & 120,00 & 120,32 & 120,65 & 120,97 & 121,30 \\
\hline Total & 396,00 & 397,07 & 398,14 & 399,22 & 400,29 \\
\hline \multicolumn{6}{|c|}{ Otros } \\
\hline Arriendo & 4800,00 & 4812,96 & 4825,95 & 4838,99 & 4852,05 \\
\hline Mantenimiento vehículo & 1200,00 & 1203,24 & 1206,49 & 1209,75 & 1213,01 \\
\hline Combustible vehículo & 1200,00 & 1203,24 & 1206,49 & 1209,75 & 1213,01 \\
\hline Total & 7200,00 & 7219,44 & 7238,93 & 7258,48 & 7278,08 \\
\hline Total Gastos Administrativos & 9969,00 & 9995,92 & 10022,91 & 10049,97 & 10077,10 \\
\hline
\end{tabular}


Gastos de ventas

Para los gastos de ventas se considera un incremento anual con la inflación del $0,27 \%$ y los detalles se encuentran en la tabla 19.

Tabla 19. Gastos de venta anual

\begin{tabular}{lccccc}
\hline Concepto & $\mathbf{2 0 1 8}$ & $\mathbf{2 0 1 9}$ & $\mathbf{2 0 2 0}$ & $\mathbf{2 0 2 1}$ & $\mathbf{2 0 2 2}$ \\
\hline Rótulos & 125,00 & 125,34 & 125,68 & 126,02 & 126,36 \\
Trípticos & 360,00 & 360,97 & 361,95 & 362,92 & 363,90 \\
Redes sociales & 240,00 & 240,65 & 241,30 & 241,95 & 242,60 \\
Uniformes de jardineros & 120,00 & 120,32 & 120,65 & 120,97 & 121,30 \\
\hline Total & $\mathbf{8 4 5 , 0 0}$ & $\mathbf{8 4 7 , 2 8}$ & $\mathbf{8 4 9 , 5 7}$ & $\mathbf{8 5 1 , 8 6}$ & $\mathbf{8 5 4 , 1 6}$ \\
\hline
\end{tabular}

\section{Gastos financieros}

Es la totalidad de intereses generados por el crédito que se solicitará. Este monto asciende a $\$ 3712,49$, el mismo que está calculado a una tasa de $11,26 \%$ anual.
El monto total a financiar es de $\$ 24465,49$ a un plazo de 3 años y el detalle se encuentra en la tabla 20.

Estados financieros

Balance general anual y flujo neto anual

Tabla 20. Balance general anual

\begin{tabular}{lccccc}
\hline Descripción & $\mathbf{2 0 1 8}$ & $\mathbf{2 0 1 9}$ & $\mathbf{2 0 2 0}$ & $\mathbf{2 0 2 1}$ & $\mathbf{2 0 2 2}$ \\
\hline Ventas & 247000,00 & 266718,20 & 286541,08 & 306469,05 & 326502,55 \\
Costo de ventas & 203348,34 & 216326,42 & 228477,42 & 242296,35 & 257829,31 \\
Utilidad bruta & 43651,66 & 50391,78 & 58063,66 & 64172,71 & 68673,24 \\
\hline \multicolumn{5}{c}{ Gastos de operación } \\
\hline Gasto administrativos & 9969,00 & 9995,92 & 10022,91 & 10049,97 & 10077,10 \\
Gasto de ventas & 845,00 & 847,28 & 849,57 & 851,86 & 854,16 \\
Gasto depreciaciones & 5382,50 & 10425,00 & 15637,50 & 20850,00 & 26062,50 \\
Gasto de constitución & 854,00 & 0 & 0 & 0 & 0 \\
Total gasto de oper. & 17050,50 & 21268,20 & 26509,97 & 31751,83 & 36993,77 \\
Utilidad antes de intereses & 26601,16 & 29123,58 & 31553,68 & 32420,88 & 31679,48 \\
Gasto financiero & 1925,82 & 1228,36 & 452,37 & 0 & 0 \\
Utilidad antes de IR y PT & 24675,34 & 27895,22 & 31101,31 & 32420,88 & 31679,48 \\
15\% Part. trab. & 3701,30 & 4184,28 & 4665,20 & 4863,13 & 4751,92 \\
Utilidad antes de impuestos & 20974,04 & 23710,94 & 26436,12 & 27557,75 & 26927,55 \\
25\% Imp. a la renta & 5243,51 & 5927,73 & 6609,03 & 6889,44 & 6731,89 \\
Utilidad del ejercicio & $\mathbf{1 5 7 3 0 , 5 3}$ & $\mathbf{1 7 7 8 3 , 2 0}$ & $\mathbf{1 9 8 2 7 , 0 9}$ & $\mathbf{2 0 6 6 8 , 3 1}$ & $\mathbf{2 0 1 9 5 , 6 7}$ \\
\hline
\end{tabular}

Tabla 21. Flujo neto

\begin{tabular}{|c|c|c|c|c|c|c|}
\hline Descripción & 2018 & 2019 & 2020 & 2021 & 2022 & 2023 \\
\hline Utilidad neta & & $\mathbf{1 5 7 3 0 , 5 3}$ & 17783,20 & 19827,09 & 20668,31 & 20195,67 \\
\hline$(+)$ Depreciación & & 5382,5 & 10425 & 15637,5 & 20850 & 26062,5 \\
\hline (-) Costo de inversión & 92752,10 & 0,00 & 0,00 & 0,00 & 0,00 & 0,00 \\
\hline Activo fijo & 77525,00 & & & & & \\
\hline Capital de trabajo & 14373,10 & & & & & \\
\hline Gastos de constitución & 854,00 & & & & & \\
\hline $\begin{array}{c}\text { (+) Recuperación } \\
\text { GDC }\end{array}$ & & 854,00 & & & & \\
\hline$(+)$ Recuperación del & & & & & & 51462,5 \\
\hline $\begin{array}{c}\text { Ac, Fi, } \\
(+) \text { Recuperación del } \\
\text { CT }\end{array}$ & & & & & & 14373,1 \\
\hline (-)Amortización & & 6194,03 & 6891,49 & 7667,46 & 0 & 0 \\
\hline$(=)$ Flujo de fondos & 92752,10 & 15773,00 & 21316,71 & 27797,13 & 41518,31 & 112093,77 \\
\hline
\end{tabular}




\section{Análisis de Factibilidad}

Con el análisis de factibilidad conocemos la rentabilidad de las instalaciones de jardines verticales y se determina las ganancias. Consideramos los siguientes métodos de evaluación:

\section{Valor Actual Neto (VAN)}

El VAN permite deducir el valor presente de un determinado número de flujos de cajas futuros originados por una inversión. El cálculo del Valor Actual Neto es esencial ya que permite valorar la factibilidad de los proyectos de inversión en un tiempo 0 , con el objetivo de ser comparados con la inversión inicial. Se ha tomado un descuento del $10 \%$ para el cálculo del VAN. Los resultados de este cálculo se encuentran detallados en la tabla 22.

Tabla 22. Valor Actual Neto (VAN)

\begin{tabular}{ccccc}
\hline Inversión total & Año & Flujo neto & $\mathbf{( 1 + 0 , 1 )}^{\mathbf{n}}$ & Flujo neto Act. \\
\hline $\mathbf{- 9 2 7 5 2 , 1 0}$ & 1 & 15772,999 & 1,100 & 14339,09 \\
& 2 & 21316,714 & 1,210 & 17617,11 \\
3 & 27797,127 & 1,331 & 20884,39 \\
& 4 & 41518,310 & 1,464 & 28357,56 \\
& 5 & 112093,766 & 1,611 & 69601,40 \\
& & & $\mathbf{5 8 0 4 7 , 4 7}$ \\
\hline
\end{tabular}

El VAN que se ha calculado para Jardines Millennium es de un monto por 58047,47 . El valor del VAN es positivo, por la cual se concluye que la empresa es rentable.

Tasa Interna de Retorno (TIR)

La TIR que se encuentra detallada en la tabla 23, es de un $25 \%$, al ser mayor a la tasa de descuento del $10 \%$ se concluye que la empresa Jardines Millennium financieramente viable.

Tabla 23. Tasa Interna de Retorno (TIR)

\begin{tabular}{cc}
\hline Año & Flujo neto \\
\hline 1 & 15772,999 \\
2 & 21316,714 \\
3 & 27797,127 \\
4 & 41518,310 \\
5 & 112093,766 \\
\hline TIR & $\mathbf{2 5 \%}$ \\
\hline
\end{tabular}

Índice Beneficio Costo ( $\mathrm{I} \mathrm{b} / \mathrm{c})$

Permitiendo conocer si es conveniente recurrir en el costo de la inversión, según los cálculos detallados en la tabla 24, por cada dólar de inversión, se recupera ese dólar y adicional \$1,63.

Tabla 24. Flujo de fondos acumulado

\begin{tabular}{cc}
\hline Flujo neto act. & Flujo acumulado \\
\hline 14339,09 & 14339,09 \\
17617,12 & 31956,21 \\
20884,39 & 52840,60 \\
28357,56 & 81198,17 \\
69601,41 & $\mathbf{1 5 0 7 9 9 , 5 8}$ \\
\hline
\end{tabular}

$$
\begin{gathered}
I(b / c)=\frac{\sum \text { Flujo Acumulado de Fondos }}{\text { Inversión Inicial }} \\
I(b / c)=\frac{150799,58}{92752,10} \\
I(b / c)=1,63
\end{gathered}
$$

$\underline{\text { Punto de Equilibrio(PE) }}$

Según la tabla 25, no se tiene perdida ni ganancia al vender 812,79 metros cuadrados de jardines verticales.

Tabla 25. Punto de Equilibrio

\begin{tabular}{lc}
\hline Denominación & Año 1 \\
\hline Materia prima & 159380,00 \\
Mano de obra & 43968,34 \\
Gastos a dministrativos & 10814,00 \\
Producción anual & 1300 \\
Costo variable unitario & 122,60 \\
\hline Total costos fijos & $\mathbf{5 4 7 8 2 , 3 4}$ \\
\hline Total costos variables & $\mathbf{1 5 9 3 8 0 , 0 0}$ \\
\hline
\end{tabular}

$P E=\frac{\text { Costos Fijos }}{\text { Precio }- \text { Costo variable Unitario }}$

$$
\begin{gathered}
P E=\frac{54782,34}{190-122,60} \\
P E=812,79
\end{gathered}
$$

26 Rev. de investig. agroproducción sustentable 3(2): 15-31, 2019 ISSN: 2520-9760 


\section{DISCUSIÓN}

Otros estudios relacionados concluyen en su investigación que implementar la jardinería vertical u otro sistema modular que lo diferencia de la competencia, representa una ventaja, además que es más practico al momento de hacer cualquier cambio o modificación, en caso de que se dañe o muera algunas de las plantas. Dentro del estudio de mercado determina que los precios son promedio en comparación a nuestros competidores ya que en Bogotá existe este sistema. En análisis técnico y financiero se concluye que el proyecto es viable en su totalidad, teniendo en cuenta aquellos aspectos en cuanto a ubicación del mismo además muestra un incremento mes a mes acumulado con proyección a cinco años. La inversión inicial es recuperada en el primer año, con la venta de 416 metros cuadrados (Arciniegas, Romero y Granados 20017).

Un estudio realizado en Guayaquil-Ecuador determina que la jardinería vertical busca satisfacer las necesidades de los clientes a través de un producto económico y eco amigable. En lo referente a lo económico financiero tiene un VAN $=\$ 14713,23$ donde el beneficio neto es mayor al monto de inversión que supera a la tasa, logrando obtener un beneficio adicional, mostrando rentabilidad del estudio financiero. $\mathrm{TIR}=28,20$ $\%$ al ver la tasa interna de retorno, se puede reflejar el resultado de VAN, haciendo visible la rentabilidad según el interés y la inversión (Ponce, 2017).

En el análisis de los dos estudios expuesto y la investigación realizada en la cuidad de Riobamba se dan un valor positivo tanto en lo técnico y económico a la implementación de la jardinería vertical ya que son innovadores, ayuda a fomentar el cuidado del medio ambiente y darle un toque novedoso al lugar donde se implemente este puede ser en la parte céntrica o arquitectónica. Además de ser una idea de emprendimientos, creando fuentes de trabajo al sector florícola.

\section{CONCLUSIONES}

El análisis del mercado potencial para la implementación de una empresa de jardinería vertical, evidenció que se cuenta con un amplio segmento de mercado, ya que los habitantes de la ciudad de Riobamba de ingresos medios y altos tienen la primicia de conservar un hábitat seguro y saludable.

Se determinó que es factible la implementación de una empresa de jardinería vertical en la ciudad de Riobamba, debido a que el servicio que brinda Jardines Millennium es aceptado ampliamente por el segmento de mercado objetivo.

Se estableció la factibilidad económica y financiera de la empresa de jardinería vertical, ya que revela una inversión primaria de $\$ 92752$ para su implementación; se estableció una tasa interna de retorno del $25 \%$ la cual es mayor a la tasa de descuento del $10 \%$ aplicada en el valor actual neto.

\section{REFERENCIAS BIBLIOGRÁFICAS}

Arciniegas, V. A. D., S. X. R. Romero, y M. J. U. Granados. 2017. Estudio de prefactibilidad para el montaje de una empresa de diseño, construcción y mantenimiento de jardines verticales y cubiertas verdes en la ciudad de Bogotá. Tesis de Grado. Escuela Colombiana de Ingeniería Julio Garavito. Bogotá (Colombia).

BCE (Banco Central del Ecuador). 2018. Informe macroeconómico. Informe Técnico. Quito (Ecuador).

Córdova, G. y M. L. Romo. 2016. Espacio urbano y actores sociales en la Ciudad de Chihuahua: ¿Mutua reconfiguración? Tijuana (Mexico): El Colegio de la Frontera Norte.

GADR (Gobierno Autónomo Descentralizado del Cantón Riobamba). 2018. Plan de uso y gestión del suelo del cantón Riobamba. Informe Técnico. Riobamba (Ecuador).

Hasan, M. M. 2013. Investigation of Energy Efficient approaches for the energy performance improvement of commercial buildings. Tesis de Maestria. Queensland University of Technology. Brisbane (Australia)

INEC (Instituto Nacional de Estadisticas y Censos). 2015. Censo de la Población y Vivienda. Datos Estadisticos. Quito (Ecuador) 
MAE (Ministerio de Ambiente del Ecuador). 2012. Sistema de clasificación de los Ecosistemas del Ecuador Continental. Quito (Ecuador): Subsecretaría de Patrimonio Natural.

Minke, G. 2012. Muros y fachadas verdes, jardines verticales. Barcelona (España): Icaria editorial.

Ponce, S. 2017. Propuesta para la creación de una empresa de instalación y mantenimiento de varios tipos de jardines verticales y otros métodos ecológicos para personas y empresas que deseen mejorar la estética y rendimiento de su inmueble. Tesis de Grado. Universidad Cátolica de Santiago de Guayaquil. Guayaquil (Ecuador).

Rodrígues, S. 2013. Análisis de factibilidad financiera de una línea de productos veterinarios. Tesis de Maestría. Universidad de Costa Rica. San José (Costa Rica).

Vargas, D. y M. M. Sanz. 2012. "El papel de los espacios públicos y sus efectos en la cohesión social: experiencia de política pública en México.” Estudios Sociológicos 30 (90): 897-914. 


\section{VII.ANEXOS}

Anexo 1. Encuesta

1. ¿Tiene usted jardín en su vivienda? Si $\quad() \quad$ No ()

¿Por qué?

Si la respuesta es SI pase a la pregunta 4. Si la respuesta es NO pase a la pregunta 2

\section{2. ¿Le gustaría tener un jardín en su vivienda? Si () No()}

Si la respuesta es SI pase a la pregunta 3 .

\section{3. ¿Qué tipo de plantas le gustaría tener en su jardín en su jardín?}

¿Por qué?

Pase a la pregunta 6.

\section{4. ¿Qué tipo de plantas tiene en su jardín?}

¿Por qué?

\section{5. ¿Dónde adquiere usted esas plantas?}

¿Por qué las adquiere ahí?

\section{Usted vive en:}

a. Casa Propia ()

b. Casa Arrendada ()

c. Departamento ()

d. Conjunto Residencial ()

e. Otros

\section{7. ¿Conoce usted sobre la Jardinería Vertical? Si （） No ( )}

Si la respuesta es SI pase a la pregunta 9. Si la respuesta es NO pase a la pregunta 8

\section{8. ¿Le gustaría conocer sobre la Jardinería Vertical? Si () No ()}

Si la respuesta es SI enseñar fotografías.

9. ¿Conoce usted una empresa en Riobamba que se dedique a la Jardinería Vertical?

Si () ¿Cuál? No()

\section{En donde implementaría un jardín vertical:}
a. Casa Propia ()
b. Casa Arrendada ()
c. Departamento ()
d. Conjunto Residencial ()
e. Otros 
11. ¿Cuál de los siguientes tipos de jardín vertical le gustaría implementar?
a. 1()
b. 2()
c. 3()
d. Todos ()
e. Ninguno () Indicar fotografías

12. Del tipo de jardín vertical que eligió. ¿Cuánto estaría dispuesto a pagar por cada metro cuadrado?
a. $\$ 180$ - $\$ 240$
b. $\$ 241-\$ 280$
c. $\$ 281$ o mas

13. ¿A través de qué medio le gustaría recibir información sobre los servicios y promociones que ofrecerá la empresa de Jardinería Vertical?
a. Radio ( )
b. Periódico( )
c. Televisión ()
d. Redes sociales [Facebook, Instagram, Whatsapp, Correo Electrónico, Twitter] ()
e. Otros

\section{Si se implementara una empresa de Jardinería Vertical en la ciudad de Riobamba} ¿estaría dispuesto a contratar los servicios de dicha empresa?
$\mathrm{Si} \quad$ ( ) No ( )
¿Por qué?

Pregunta 11. Tipos de jardín vertical

1. Sistema Modular Simple

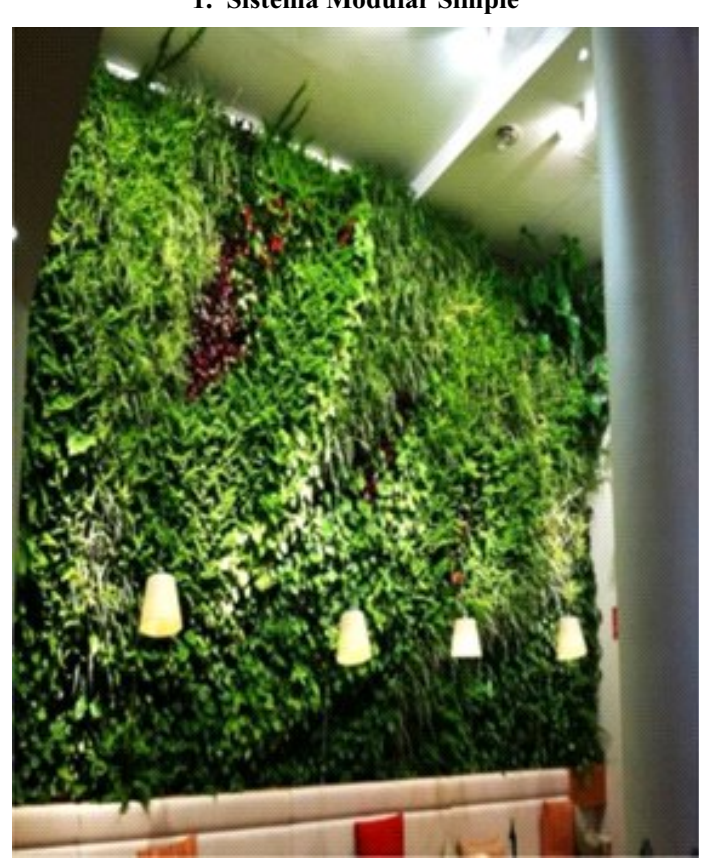

30 Rev. de investig. agroproducción sustentable 3(2): 15-31, 2019 ISSN: 2520-9760 
2. Sistema Modular Complejo

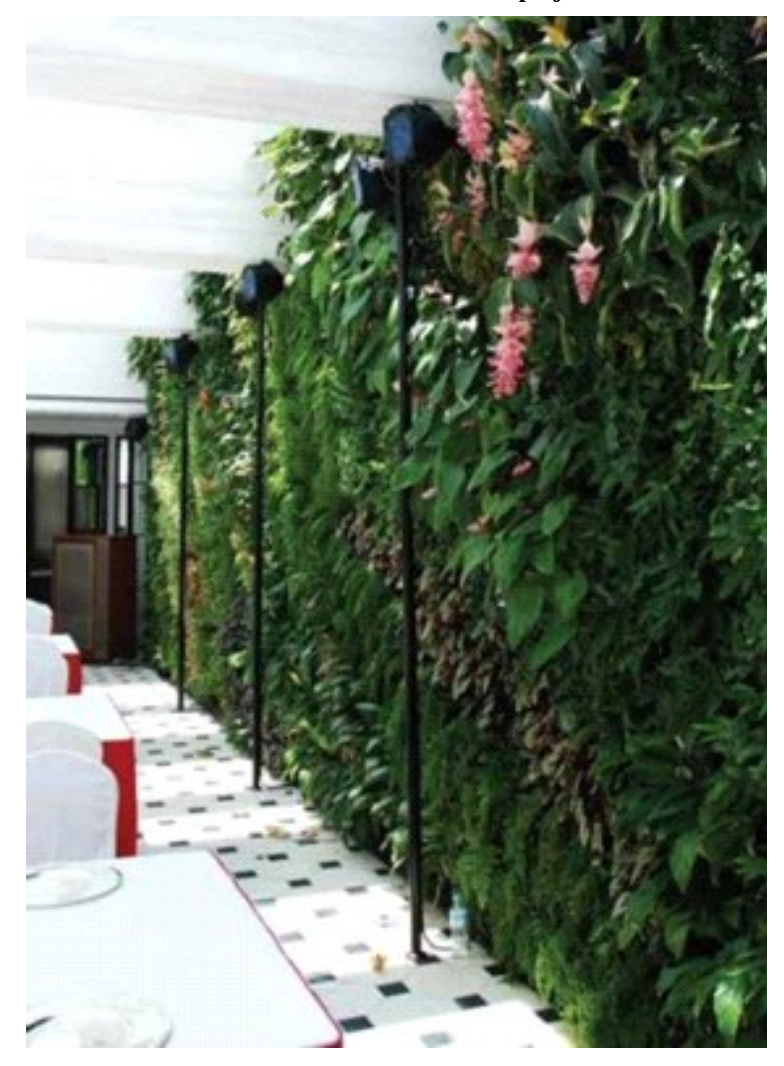

3. Le mur vegetal (Patrick Blanc)

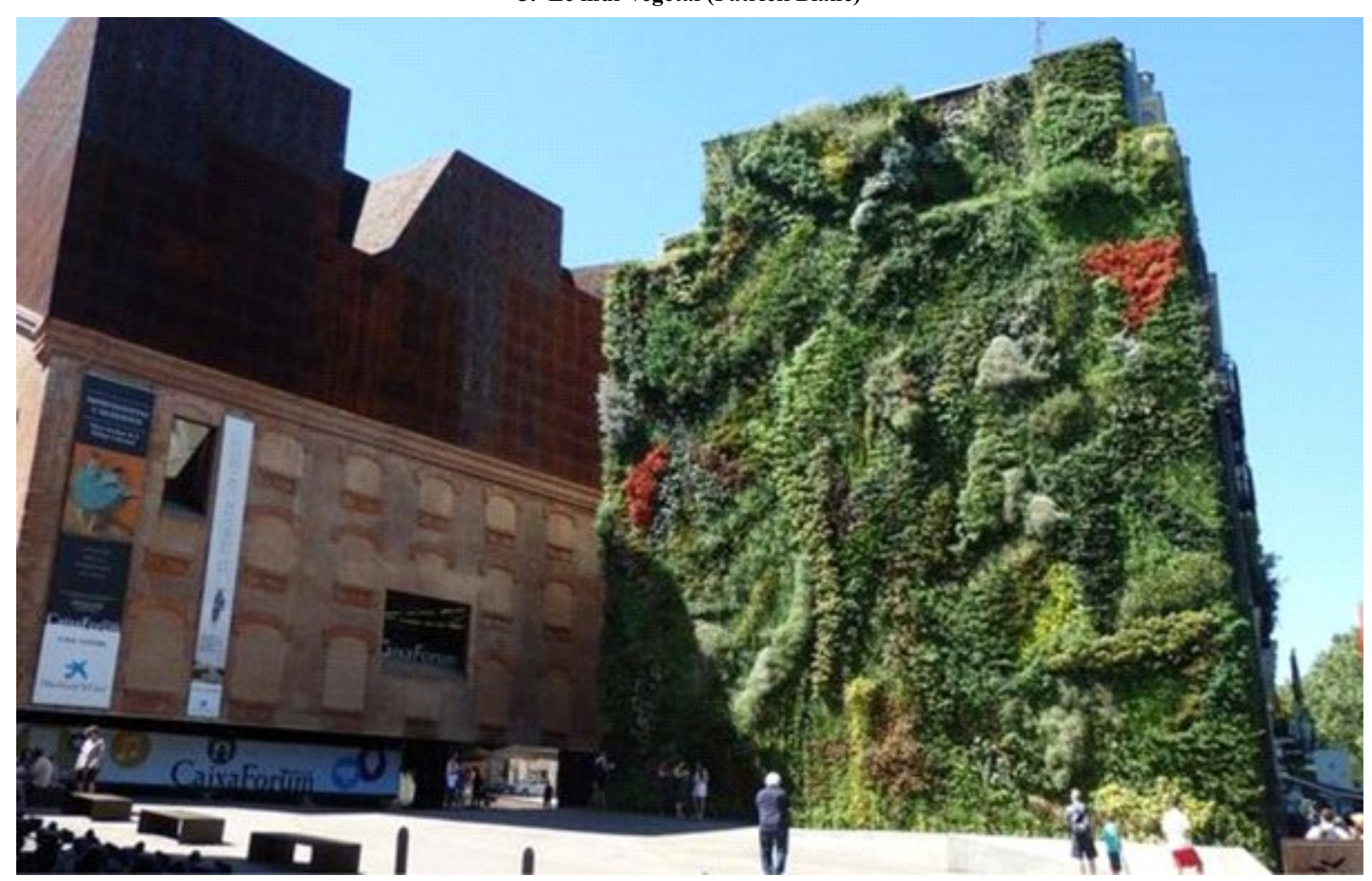

